\title{
Repensar la organización disciplinar en la universidad, una crítica aproximada desde el enfoque de la complejidad
}

\author{
Dr. Raúl Jáuregui Mercado \\ Universidad La Salle (Perú) \\ rjauregui@ulasalle.edu.pe
}

Recepción:24/05/2021

Aceptación: 17/06/2021

\section{Resumen}

La crítica propuesta pone en cuestión a la organización del conocimiento en la universidad como institución creadora y re-creadora de conocimiento, buscando contextualizarla en el seno de la sociedad. Para ello se apela al enfoque de la complejidad, como una de alternativa a través de la cual se realiza una somera revisión retrospectiva de los principios que dieron lugar a la organización disciplinar formalizada en la estructura de la universidad. Se discute sus implicancias y sus limitaciones para enfrentar los problemas que aún aquejan a la humanidad. A través de los principios asociados a la complejidad como son el principio sistémico, hologramático, retroaccional, recursivo, la auto organización, lo dialógico; y la reintroducción, se han ensayado algunas reflexiones ciertamente discutibles que pueden contribuir a aprovechar la potencia y acto de los saberes disciplinares en un intento de diálogo fecundo a través de mecanismos tipo interfases sinápticas. Las meditaciones anotadas sugieren que es posible intentar repensar la organización del conocimiento respetando las disciplinas, sugiriendo al mismo tiempo crear respuestas desde un plano filosófico, epistemológico y metodológico, que puedan responder a la complejidad de las realidades que se encuentran traslapadas, en cuyo laberinto también se encuentra la universidad.

\begin{abstract}
The proposed criticism calls into question the organization of knowledge in the university as an institution that creates and re-creates knowledge, seeking to contextualize it within society. For this, the complexity approach is appealed, as an alternative through which a brief retrospective review of the principles that gave rise to the formalized disciplinary organization in the university structure is carried out. Its implications and limitations to face the problems that still afflict humanity are discussed. Through the principles associated with complexity such as the systemic, hologrammatic, retro-actional, recursive principle, self-organization, the dialogic; and reintroduction, some admittedly debatable reflections have been tested that may contribute to harnessing the power and action of disciplinary knowledge in an attempt at fruitful dialogue through synaptic interface-type mechanisms. The an-
\end{abstract}


notated meditations suggest that it is possible to try to rethink the organization of knowledge while respecting the disciplines, suggesting at the same time creating responses from a philosophical, epistemological and methodological level, which can respond to the complexity of the overlapping realities, in which labyrinth also the university is located.

\section{Palabras clave}

Conocimiento, complejidad, organización, universidad, sociedad.

\section{Key words}

Knowledge, complexity, organization, university, society.

\section{Propósito de la cuestión}

El presente ejercicio reflexivo propone una crítica aproximada desde un enfoque de la complejidad a la forma de organización tradicional del conocimiento en el seno de la universidad, sabiendo que se le ha atribuido ser una institución creadora de conocimiento y formadora de profesionales aptos para enrolarse en el mercado laboral. Por supuesto que hay otras causas finales (en términos aristotélicos) que se le atribuyen, como es encargarle la preservación de la cultura, entre otras colindantes.

Acometerla no solo es un compromiso en su dimensión ontológica, epistemológica; sino también moral. Si bien Morin (2002), alega que ello será posible solo mediante una reforma en el pensamiento, sin embargo, se necesita ir más allá de la metáfora de solo repensar, que de por si, encierra una pretensión generalista, sino fundamentalmente en orientar una crítica evitando usar el concepto "complejidad" como una especie de muletilla aplicable a toda situación.

La universidad fue inventada en el siglo XII, inicialmente como escuelas catedralicias y escuelas monásticas. Su organización de origen es esencialmente disciplinar lo que se mantiene hasta hoy. El conocimiento ha sido dividido en disciplinas para ser impartido mediante una organización facultativa.

La organización disciplinar especializada logró importantes resultados en diversos campos de la ciencia así como de la tecnología, cuyos frutos le han permitido al ser humano alcanzar una mejor calidad de vida, satisfacer sus necesidades, y principalmente a una mayor democratización del conocimiento.
Al propio tiempo, con todo ese caudal de conocimiento científico y tecnológico el ser humano aun no ha logrado resolver los problemas que ahora le aquejan. En términos de Prigogine (1983) el conocimiento científico logrado por el ser humano no ha sido capaz de dar respuesta al azar, a las fluctuaciones, a las bifurcaciones y ampliaciones. Vilar (1997) agrega que tales problemáticas están presionando a las universidades a salir del círculo cerrado de las disciplinas, para aceptar con realismo que los problemas tienen una complejidad tal que no podrán ser tratados desde el reductivo mundo de las disciplinas separadas.

Si bien los problemas que aun aquejan al ser humano tienen complejidad, las respuestas, desde el conocimiento científico y tecnológico, no tendrían que basarse únicamente en un conjunto de conocimientos categorizados sea como "teorías complejas" o "ciencias de la complejidad" sin antes haber reflexionado si ello es realmente posible. Lo expresado tanto por Progonine como por Vilar, es apenas una explicación parcial y riesgosa, en especial cuando se sugiere la necesidad implícita de incorporar la complejidad (ciencias de la complejidad y/o pensamiento complejo) con el fin de diluir a las disciplinas, sin que se cuente con experiencias o praxis en los que se haya aplicado y demostrado, vía contrastabilidad, alguna teoría o ciencia de la complejidad, no solo en un nivel de reflexiòn filosófica y epistemológica, sino también metodológica.

Por ello, parece importante proponer una alerta crítica para evitar caer en un cerrado dogmatismo que puede llevar a una confusión entre la complejidad del ser de las realidades caracterizada por sus múltiples dimensiones, planos de referencia y multi factores intervinientes; con la pretensión de dar respuestas basadas en las "ciencias de la complejidad" (si estas son epistemológicamente viables) o del "pensamiento complejo" como una especie de cura para todos los males.

\section{Enfoque para una crítica alternativa}

A partir del reconocimiento de la situación planteada, se intentará ensayar un conjunto de reflexiones, sin que en rigor se constituyan en la única respuesta. Dicho intento se realizará desde el enfoque de la complejidad, no tanto desde una causación o causalidad discutido ampliamente por Bunge (1997), sino intentando salir a la orilla del río para mirar aguas arriba y aguas abajo, si vale la metáfora.

Partiendo de la idea o concepto de complejo que en latín tiene acepciones como: plecto, plexi, complector, plexus; que significan: tejido, trenzado, enredado (Ar- 
doino, en Morin, 2000); se puede agregar entretejido, para comprender la variedad de la existencia de realidades traslapadas.

En una breve retrospectiva, René Descartes, en el discurso del método, sitúa los orígenes de una forma de organización del conocimiento científico sustentados en un conjunto de principios que rigen hasta hoy. Su institucionalización daría lugar al llamado pensamiento cartesiano. Morin (2000) ensaya una explicación de tales principios.

Así, el principio de orden, aclara que no se refiere a "determinismo", ya que el orden abarca al determinismo, representado por el orden perfecto de la naturaleza, sin cuestionar las razones de dicho orden; las inexactitudes, desórdenes y lo aleatorio, que identificados como problemas, estos eran más atribuibles a la insuficiencia de conocimientos que al omnisciente orden.

El principio de disyunción o separación del conocimiento objetivo en materias unas de otras, el sujeto que busca conocer del objeto por conocer. Ello condujo al principio de especialización, la que a su vez produjo la posterior organización del conocimiento por disciplinas. Cada disciplina encerrada en sus propias fronteras, cuando muchas ideas nuevas aparecieron precisamente en las zonas inciertas ubicadas en las borrosas líneas de sus fronteras. Descartes sugirió que para resolver las dificultades habría que dividirlas para resolverlas mejor, y ordenar el pensamiento conociendo primero los objetos sencillos, para ir de manera progresiva al conocimiento de objetos de mayor complejidad.

El principio de la validez absoluta de la lógica clásica considerada como deductiva, inductiva, identitaria, que atribuye un valor de verdad a la inducción, la deducción, por lo cual, toda contradicción debería ser eliminada, la identificación o presunción de un error implicaría abandonar un razonamiento. De esta manera queda instalado el principio de la causalidad lineal.

Estos principios cartesianos se han constituido en los mecanismos metodológicos que dominan la ciencia hasta ahora y sobre los cuales se ha decidido organizar el conocimiento generado e impartido en la universidad. Desde un enfoque de la complejidad, el pensamiento que une debería no tanto reemplazar a la causalidad unilineal y unidireccional, sino complementarla con una especie de causalidad en bucle y multirreferencial, rompiendo en cierta forma con los axiomas de la lógica clásica, por medio de una dialógica capaz de concebir unas nociones complementarias y antagónicas que posibiliten una integrada comprensión de las partes en y con el todo.
Dicho bucle multirreferencial permitiría, más que una explicación, una comprensión. La primera es necesaria para un ejercicio intelectual, objetivo, un juzgar racional como señala Husserl (1975), pero insuficiente para la comprensión humana, subjetiva. Sobre ello, cabe contraponer en un sentido aristotélico, un proceso reductivo que se genera entre la realidad como el ser objeto de estudio, y la escritura con el que se argumenta alguna región del ser, pues, de alguna forma comprender es comprimir, por lo tanto, las explicaciones de los fenómenos caen en un proceso reductivo, pues, la realidad del ser se reduce en el pensamiento, el pensamiento se reduce en el lenguaje, y el lenguaje de reduce en la escritura.

Moreno (2002) agrega que un intento de comprensión de la complejidad, muchas veces se asocia con lo complicado. En ese sentido sugiere que lo complicado debe ser o puede ser entendida tanto en sentido psicológico vinculado a una especie de incapacidad de comprensión que supera la capacidad intelectual, así como la posibilidad de traducir la realidad en pensamiento y luego en lenguaje que trascienda al ser.

Reynoso (2009) y García (2006) coinciden en advertir que los argumentos expuestos en toda la obra moriana no hay nada que parezca una teoría operativa lista para ser aplicada. La advertencia señalada no invalida la posibilidad, no de un aterrizaje forzoso, sino de una meditada reflexión desde y entre disciplinas para procurar espacios de diálogo, como es unir los conocimientos (Morin, 2000), que conduzcan también a un diálogo epistemológico y metodológico, aun pendiente.

Para intentar una aproximación metodológica desde un enfoque de la complejidad, es posible aplicar un conjunto de principios como son: el principio sistémico, el principio hologramático, el principio del bucle retroactivo, el principio del bucle recursivo, el principio de la auto organización, el principio dialógico; y el principio de la reintroducción (Morin, 2002).

Por el principio sistémico se puede lograr una comprensión del ser de las realidades, desde una comprensión de las partes, hacia la comprensión del todo, y del todo hacia las partes. Sin embargo, aquí se presenta un dilema de orden filosófico y epistemológico, en relación a una ontología del ser como totalidad, en su extensa infinitud, ya que el todo en términos de conocimiento no es lo mismo que el todo del ser-objeto, el conocimiento se queda como acto cuya potencia implica un movimiento que reduce la ignorancia sobre el ser, en tanto objeto de estudio.

Ahora bien, lo sistémico que se opone a un reduccio- 
nismo disciplinar (en tanto explicación parcial de los objetos que realiza cada disciplina) deviene en una especie de "sordera especializada" que se resume en dos opuestos, a saber, "el todo es más que la suma de sus partes", que trasciende las cualidades y propiedades que emergen por la interacción e inter penetración de ese todo con y en sus partes; y el "todo es menos que la suma de sus partes", cuando las propiedades o cualidades del ser del objeto son inhibidas por las explicaciones parciales de cada disciplina.

En relación al principio hologramático, se precisa que este pone en evidencia la aparente paradoja de las organizaciones complejas donde no sólo la partes se encuentran en el todo sino donde el todo está inscrito en las partes. Este principio está inspirado en el holograma en el que cada punto contiene casi la totalidad de la información del objeto que representa. Así, por ejemplo, la totalidad del patrimonio genético se encuentra en una célula. Por extensión se podría afirmar que el patrimonio genético de una cultura se encuentra en sus instituciones y en el individuo.

En cuanto al bucle retroactivo, cuyo marco conceptual de origen fue propuesto por Norbert Wiener. Dicho principio constituye una autorregulación de los procesos. Tal mecanismo va mucho más allá de lo que se conoce como causalidad lineal, es decir, una causa actuando sobre un efecto, y el efecto actuando sobre la causa; en una especie de regulación. Aquí se puede precisar, que, teniendo un organismo vivo un conjunto de procesos reguladores, éstas se fundan en múltiples retroacciones, una "homeóstasis compleja".

Como lo expresa Johansen (1986), la retroacción lineal está asociada con la retroalimentación solo negativa que es de orden correctivo. Sin embargo, al observar a los organismos vivos: célula, cuerpo, sociedad; dado a su complejidad, la retroacción negativa es reductora de sus emergencias (el todo es menor que la suma de sus partes), en cambio, se puede observar que se producen retracciones de orden positivo cuando, por ejemplo, en términos de organización, se mantienen las acciones que emergen generando la necesidad de modificar los objetivos; y la retroacción conocida como desviación - amplificación que encierran procesos de inter conectividad e inter penetrabilidad mutuas que amplifican un efecto inicial. Un ejemplo explicativo de esta naturaleza está en el llamado efecto mariposa.

El bucle recursivo es un principio que supera la noción de regulación por la de autoproducción y auto organización. Los productos y los efectos son ellos mismos productores y causantes de lo que los produce. Las personas como individuos producen la sociedad que de- viene en una cultura, a su vez, la sociedad como un todo emergente produce el sello de la humanidad en cada individuo, por ejemplo, a través del lenguaje, las costumbres, las tradiciones, las normas, el conocimiento; una cultura.

Por el principio de auto-eco-organización se reconoce a los seres vivos como auto organizadores que sin cesar se auto producen y por lo mismo consumen energía para sostener su autonomía. Como quiera que su capacidad de autonomía depende de sus capacidades de interrelación con sus entornos próximos y remotos hay la imperiosa necesidad de extraer de su medio, energía e información que modele su organización, por lo tanto, su autonomía es inseparable de dicha dependencia e inter dependencia; es regenerativa. A ello se suma lo expresado por Maturana y Varela (1990) quienes siguiendo a Foerster y Von Neumman, crearon el concepto de autopoiesis, equivalente a autoproducción como la base de la auto organización.

El principio dialógico se expresa en la unión de dos nociones o principios que deben excluirse mutuamente, pero que a la vez son indisociables en una misma realidad. Heráclito expresaba: "vivir de muerte, morir de vida", en una metafórica alusión de la dialógica del ser que al nacer comienza a morir, que a su vez genera su devenir efímero, desde una inmanencia hacia su trascendencia.

Bajo las formas más diversas, la dialógica entre el orden, el desorden y la organización, a través de innumerables inter retroacciones, están constantemente en acción en las dimensiones físico, biológico y humano. La dialógica permite asumir racionalmente la inseparabilidad de unas nociones contradictorias para concebir un mismo fenómeno. Se diría que los individuos son como corpúsculos autónomos desde un cierto punto de vista. En el seno de las dos continuidades que son la especie y la sociedad; cuando se considera la especie o la sociedad, el individuo desaparece; cuando se considera al individuo, la especie y la sociedad desaparecen. Por lo tanto, la dialógica debe asumir los dos términos que tienden a excluirse mutuamente. La dialógica, de esta manera, rompería con el "tercero excluido" uno de los principales axiomas aristotélicos muy vigentes en el pensamiento actual.

La reintroducción del conocedor en todo conocimiento, es un principio que opera la restauración del sujeto en el propio conocimiento que generó. En el entendido que todo conocimiento es una reconstrucción/traducción realizada por el cerebro humano en una cultura o tiempo dados. Desde una perspectiva histórica de la ciencia, que, hasta cierto punto es relativista, cada 
época tiene su propio paradigma, por lo tanto, no se pueden comparar. Aquí se aplica el principio de inconmensurabilidad propuesto por Kuhn (1972).

En suma, una reforma desde un enfoque de la complejidad es de naturaleza paradigmática y no programática, implica la aptitud para organizar el conocimiento en su trama y entre tejido, considerando su potencia, movimiento y acto a través de su disciplinariedad, su inter disciplinariedad, su multidisciplinariedad y su probable transdisciplinariedad. Hay que considerar que este ejercicio entre disciplinas no debe significar trangredir fronteras de manera arbitraria, ello para evitar andaduras indisciplinadas y antidisciplinarias.

\section{A modo de discusión para la reflexión}

Con base en el enfoque de referencia que es muy insuficiente para intentar una crítica, se anotan algunas reflexiones que van en el entendido del bucle universidad-sociedad-universidad.

La organización del conocimiento en disciplinas instituida en la universidad tiene como el principal agente a la cátedra y a quien la ejerce. Como expresa Morin (1992) en las sociedades teocráticas de la antigüedad, los saberes cosmológico, mágico, mitológico y religioso estaban concentradas en los mismos espíritus y en la casta de sacerdotes y magos, tanto así que las verdades consideradas supremas no eran accesibles, se requería de una iniciación muy prolongada. En dicho contexto, lo que hoy se llama pueblo, éste se desenvolvía inmerso en prácticas transmitidas de generación en generación, según cada oficio.

Luego, el saber moderno fue asumido por la clericatura de la iglesia, a continuación, el término clérigo se laicizó y profesionalizó. A la antigua clericatura le sucedió la intelligentsia, los intelectuales. En tal sentido, la intelligentsia comprende a las disciplinas Ilamadas carreras o profesiones que producen y reproducen el saber. Incluso se generó una clasificación, esto es, los saberes a cargo de enseñantes e investigadores, las ideas a cargo de filósofos, las formas a cargo de artistas, arquitectos, diseñadores, los que dependían de las ideas como los abogados, el saber experto o de la concepción ejercidas por los ingenieros. Así, las actividades y saberes asociados a la artesanía, la pesca, la caza; entre otras no formaban parte de la élite de la intelligentsia.

Se puede notar que en los albores de la organización del conocimiento se siguieron los principios cartesianos que han implicado la división "natural" de la ciencia en disciplinas, cada cual, con su objeto de estudio, encerrada en sí misma, endogámica y reducida al conocimiento de una parcela de la realidad, generándose de esta manera lo que se suele llamar, "sordera especializada".

Por otra parte, la revolución industrial marcada por la especialización en la producción en serie de bienes y luego de servicios, lograron consonancia con la forma de organización del conocimiento disciplinar, algo así como la instalación de una demanda y oferta de conocimiento. El desarrollo de una nueva cultura científica va a determinar no solo una ruptura epistemológica entre filosofía y ciencia, sino también una ruptura ontológica entre cultura científica y cultura humanística. Se había producido una especie de taxonomía separatista del conocimiento con diversas acepciones, sea ciencias duras, ciencias blandas, o ciencias formales, ciencias fácticas; entre otras.

La oferta de conocimiento organizada en la universidad adoptó también una fragmentación en torno a facultades, y luego aparecieron los Ilamados departamentos académicos como espacios congregantes de pares cultivando disciplinas en común.

Berg (1977) ya explicaba la evidencia empírica sobre el particular, al reafirmar que la unidad de adscripción básica de los sistemas académicos está organizada en torno a la disciplina. Como se ha observado en Suecia, incluso después de grandes dosis de modernización, el grupo de miembros más importantes está compuesto por docentes e investigadores, organizados en subsistemas de acuerdo con la, disciplina (departamento y similares), y su competencia fundamental así como su identidad profesional se derivan básicamente de la disciplina. Ésta también determina sus contactos nacionales e internacionales.

En efecto, el mismo Berg (1977) agrega que las disciplinas determinan en gran parte la división del trabajo en los establecimientos y confieren contenido a las divisiones. Cada una detenta algo que se aproxima a un monopolio local sobre el conocimiento especializado respecto de una operación concreta. Y este proceso se agudiza conforme las disciplinas se profesionalizan y fortalecen su autonomía al enfatizar la certificación, las calificaciones y las jurisdicciones con miras a trazar sus fronteras con claridad.

Cada unidad de disciplinas de un establecimiento tiene una primacía proclamada y evidente en el ejercicio de una determinada tarea sustantiva en la "trinchera" académica. Es inherente al estatus de un departamento (en Inglaterra) el hecho de ser una autoridad en su campo de conocimiento. La autoridad legítima de individuos y grupos se ejerce en todas las áreas del saber, cada una de las cuales tiene un papel sustantivo en la 
docencia, la investigación y otras formas de manejar el conocimiento. Los miembros académicos de un departamento de física cumplen esta función, y ningún otro grupo en la institución o el sistema en general puede afirmar legítimamente que su conocimiento de la física o de la operación del campo de la física es comparable a la de los físicos. En todos los campos sucede lo mismo. Las características de los grupos integrantes básicos condicionan todos los aspectos importantes de la organización. Los rasgos especiales de los grupos académicos hacen de las universidades algo distinto de cualquiera otra organización unitaria; en virtud de estos rasgos, el control de tipo colegiado no es un accidente, y se requiere un vocabulario peculiar para describir oficios y gremios, federaciones y conglomerados; para revelar las realidades de la organización académica que se ocultan cuando son designadas con la terminología común de la vida organizacional.

Berg (1977) concluye que, unidades semejantes son legión e incluso dominan en Europa y América Latina. Los patrones de organización disciplinar franceses e italianos ejemplifican esta situación. Las facultades europeas tienen personal, clientela y recursos propios, pese a su ubicación formal dentro de sistemas públicos nacionales y regionales que aparentemente tenderían a cohesionarlas. Dichas facultades han sido tan independientes que nunca ha habido necesidad de agruparlas físicamente. De ahí que aparezcan dispersas en los centros urbanos como algunas universidades norteamericanas en las que la escuela de medicina se encuentra separada de la de administración o agronomía por un río importante o incluso está ubicada en otra ciudad.

Clark (1991) clarifica lo descrito, al mencionar que la autonomía de las facultades tiende a ser muy grande en las universidades compuestas primordialmente de unidades profesionales especializadas, rasgo común a la mayoría de las universidades de la Europa continental y de aquellas que las han imitado. Con frecuencia, se menciona esta característica al analizar los temas de autoridad e integración. También menciona que en Estados Unidos tienden a ser muy autónomas las escuelas o facultades centradas en una sola profesión. De hecho, dichas unidades a menudo se bastan a sí mismas. En las universidades privadas, se les exige que obtengan financiamiento propio; en las universidades estatales, los subsidios aparecen como rubros fijos y obligatorios de los presupuestos estatales; y tanto en las universidades públicas como en las privadas, las escuelas profesionales pueden llegar a convertirse de hecho en brazos educativos de alguna agencia gubernamental nacional, como es el caso de la absoluta dependencia de las escuelas de medicina, en universidades importantes, respecto al Servicio de Salud Federal en Estados
Unidos, situación a partir de la cual se acuñó el término de "universidad de subsidio federal".

Estas evidencias que reflejan una institucionalización organizativa disciplinar fuertemente canonizada, limita el potencial sinérgico del telos del conocimiento científico. La forma de socialización del conocimiento, fundamentalmente disciplinar, estaría contribuyendo a reforzar la vigencia de una sociedad incomprendida, una sociedad cuyas problemáticas y cuyas demandas han rebasado a una oferta de un conocimiento orgánicamente parcelado, incapaz de dar respuestas integrales coherentes a las dimensiones de las múltiples realidades, ello, a pesar de los esfuerzos de iniciativas de acciones inter y multidisciplinarias, pero con una débil conexión en su potencia y acto.

A partir de ello viene una primera reflexión, se puede comprender que la época reciente, muy fecunda en conocimiento, sea al mismo tiempo trágica para el propio conocimiento. Pues, hay en la cultura humanista una especie de degradación, las vallas de las fronteras levantadas son muy altas, ya no reciben el oxígeno de los conocimientos científicos, las humanidades y las ciencias sociales no poseen la parafernalia instrumental que les permitan grandes avances como sí ocurre en las otras parcelas de conocimientos considerados como "duros".

Del mismo modo, en la cultura científica, cada vez más enraizada a un conocimiento, por un lado, matematizado y manipulador y, por otro lado, parcelarizado y disjunto, se produce una especie de rarefacción de la reflexión. Considerando que la reflexión une un objeto en particular con el conjunto del que forma parte, y este conjunto al sujeto que reflexiona, resulta, por decir lo menos, imposible reflexionar sobre los saberes parcelados divididos en trozos de conocimientos que finalmente, en la praxis, se anulan entre sí. Una reflexión basada en el reduccionismo intelectual es hasta cierto punto inmoral.

Ahondando en evidencias, el conocimiento en el actual estado de organización no puede reflexionar sobre sí mismo, ya que el cerebro del que procede es estudiado en los departamentos de neurociencias; el espíritu que los constituye es estudiado en los departamentos de psicología; la cultura de la cual depende es estudiada en los departamentos académicos de sociología; la lógica que lo controla es estudiada en un departamento de filosofía. Dichos departamentos, a semejanza de los conjuntos habitacionales unifamiliares, o departamentos estanco son institucionalmente no comunicantes. En razón de ello, el conocimiento científico no se conoce a sí mismo, ignora la otredad de la parcela de al lado, 
no conoce su papel en la sociedad, no conoce el sentido de su devenir, ignora las nociones de consciencia y de intersubjetividad, privándose del derecho a la reflexión, que supone la auto observación de un sujeto consciente que intenta conocer su conocimiento. Ahora se puede comprender que el saber, que tradicionalmente era producido para ser reflexionado, meditado, pensado, discutido, incorporado, está cada vez más condenado a ser ventilado en las rúbricas especializadas y a ser acumulado en los bancos de datos. No existe solidaridad entre y con las disciplinas, al estar encerradas en la caverna de un oscurantismo intelectual, esas aguas riegan a la sociedad, la cual también aparece en la misma caverna, encerrada, postrada y condenada a una posible auto extinción.

Por otra parte de ninguna manera se debe colegir que el problema está centrado en la naturaleza de las disciplinas, sino fundamentalmente en su organización para la praxis. La complejidad de las realidades, exigen otras alternativas de organización de las disciplinas a través de disciplinas interfases que simulen una especie de redes neuronales. Estas no se pueden dar si no hay neuronas. Una praxis inter, multi o transdisciplinar no se puede dar sin disciplinas.

Ahora bien, en el contexto de los principios esbozados desde un enfoque de la complejidad, un re-pensar de la universidad implicaría, desde lo sistémico, implementar una especie de vasos comunicantes o un cuerpo calloso que una los hemisferios multi cerebrales integrados por la diversas disciplinas. La interconectividad e interpenetración sustantiva, tendrá que navegar por las aguas que conecten archipiélagos de conocimientos, a través del diálogo de saberes. Es necesario insistir que no se está contra de la disciplina, ella, es una condición necesaria pero no suficiente para una reclamada solidaridad cognitiva e intelectual, no impuesta, sino una solidaridad emergente a las necesidades, demandas subyacentes y problemas de la sociedad. Es necesario, en un plano metodológico, pasar a una praxis de lo ya escrito en torno a posibilidades de diálogos de saberes con respeto a los cánones epistemológicos que el conocimiento científico exige.

En cuanto a la transdisciplinariedad, Nicolescu (1996) advierte que absolutizar la naturaleza de la transdisciplinariedad en su relación con la disciplina, la interdisciplina y la multidisciplina es extremadamente peligroso ya que la transdisciplinariedad sería vaciada de todo su potencial contenido y su eficacia en la acción reducida a la nada.

Es importante destacar que una reforma organizativa del conocimiento basada en dichos niveles evitará la inhibición producida por la organización parcelada del conocimiento, por el contrario, su interconectividad hará que aparezcan nuevas alternativas acotadas a más regiones del ser, una especie de nodo emergente sinérgico.

La aplicación del principio hologramático, asociado a una imagen que encierra una especie de totalidad, en el que como dice Barthes (1986), implican un nivel informativo y un nivel simbólico sintetizado en un corpus que da cuenta de algo sobre el ser, pero que ese algo representa, siendo parte, a una región de esa totalidad del ser.

Desde un punto de vista biológico, un ser vivo se ubica en su contexto a través de su sistema nervioso gobernado por el cerebro. El cerebro tiene el potencial de "ver" totalidades, o al menos percibir totalidades cuando se enfoca en una región del ser. Sin embargo, cuando se le entrena para "ver" totalidades reducidas, se produce una especie de ceguera, en este caso, de tipo intelectual. No se es capaz de ligar una microestructura con una macroestructura (Kanizsa, 1986). Este punto es crucial, una visión holónica implicaría una solidaridad intelectual, una convergencia de saberes. La disciplina seguirá siendo una condición necesaria, la emancipará y la integrará siendo a la vez parte y todo en relación al conjunto de otras disciplinas. Las fronteras abiertas posibilitarán adecuar extrapolaciones a diversas escalas en el seno de la sociedad, en un sentido aristotélico, la integración de los contrarios: universal y particular.

El conocimiento, que es el contenido, tiene un continente formal que es la forma cómo se lo organiza. La forma cartesiana disyuntiva inhibe la posibilidad de aplicar una multi retroacción que mayormente se reduce a una regulación correctiva. La disciplina por sí sola no puede retroaccionar la totalidad, más allá de una regulación correctiva. Aun cuando sea conveniente (en términos de gestión), en una organización funcional es imposible una multi retroacción al efecto de conjunto. Está más allá de las fronteras de la disciplina. Retroaccionar una parte del cuerpo, no significa retroaccionar al cuerpo. El que una disciplina retroaccione una región del ser acotado a su jurisdicción, no implica ni puede retroaccionar a más regiones del ser. Sin embargo, ello no significa que se inhiba las retroacciones que ocurren en el seno de ellas, que como partes, tienen a su vez el comportamiento de un todo.

Si el ser tiene un origen ontológico que en su génesis responde a las cuatro causas precisadas por Aristóteles, a saber: formal, material, eficiente y teleológica o final; $y$, a la vez su devenir va de la potencia al acto, entonces, tiene una capacidad recursiva para auto organizarse y 
auto producirse. Ello constituye una complejidad cuya inmanencia trasciende de manera limitada a nivel disciplinar. En ese mismo sentido, cuando el ser presenta desviaciones y fluctuaciones sea como problemas estructurales y no estructurales, como ocurre en el seno de la sociedad, aparecen dificultades en su causa material y eficiente, que inhiben su telos. Una intervención solo disciplinar posee una comprensión limitada de esa complejidad, por lo tanto, la ciencia regionalizada y especializada fracasará una y otra vez en proponer respuestas integrales a la complejidad de las problemáticas situadas en las múltiples realidades.

Las múltiples realidades traslapadas entre sí, representadas en la cultura y las culturas devienen como producto de la relación entre la naturaleza dada y la intervención de la inteligencia humana para crear un hábitat. Se comportan como un sistema vivo, no colectivo, sino en un proceso de auto organización que en términos de Schuldt (2005) propenden a buscar una autodeterminación, autosuficiencia y un autocentramiento. Desde un enfoque de la complejidad, estas aspiraciones están relacionadas con el principio de auto organización.

Considerando que la universidad es en esencia creadora de conocimiento con cuyo regadío se fertiliza a la cultura, dándole una capacidad auto productiva, que en términos de Maturana y Varela (1990) constituye lo que ellos llaman sistemas autopiéticos. A partir de ello, la reflexión va asociada con la necesidad de asumir una crítica de cómo el conocimiento que se produce, al ser insertado en la sociedad como práctica social en los diversos espacios de convivencia, también tiene consecuencias cuyos efectos vuelven al seno de la universidad, cuando se reciben a personas que tienen una génesis impregnada de la sustancia cultural recibida en los valores que transmite la familia, así como en su formación básica. Somos productores producidos de manera indefinida. Desde una racionalidad, casi todas las decisiones se toman en base al conocimiento científico, tecnológico y también el conocimiento no científico. Las consecuencias de esas decisiones se manifiestan en diversos resultados, muchos considerados como satisfactorios en la medida en que contribuyen a la calidad de vida de los seres humanos y su entorno; y también consecuencias que afectan a la calidad de vida y al entorno de los seres humanos y de los seres vivos en general. La autopoiesis y la adaptación son condiciones necesarias para la existencia de los seres vivos; la primera es la organización mínima de lo vivo, mientras que la segunda es la congruencia entre el ser vivo y el entorno.

Uno de los principales valores que caracterizan a la uni- versidad es que en su seno se crean las condiciones para un ejercicio autónomo de la libertad expresada en la libertad de cátedra en cuya acepción se involucra a la comunidad en pleno y no solo al catedrático. Por lo tanto, la denominada tolerancia hacia y entre posiciones es un llamado a mantener un apertura que evite la discriminación de orden intelectual. Por otra parte, la argumentación que es el centro de lo que se hace en la academia y que forma parte de los resultados de la producción de conocimiento, se ha construido en base a una lógica, principalmente aristotélica en el que se reconocen algunos axiomas clásicos como son el principio de identidad, el principio de no contradicción, el principio del tercero excluido y el principio de bivalencia.

Desde un enfoque de la complejidad el que más se pone en tela de juicio es el principio del tercero excluido que afirma que, dados un enunciado cualquiera y su negación, uno de ellos, y sólo uno, es verdadero; es decir, las dos proposiciones no pueden ser verdaderas sobre un mismo asunto u objeto. Frente a ello surge el principio diálogico que buscan convertir al tercero excluido en tercero incluido, es decir, que dada una proposición verdadera, su contrario no sería el error o la falsedad, sino otra verdad, en términos de isomorfismo, casi una cuántica proposicional.

En esa perspectiva, el principio dialógico, desde la complejidad, pretende romper los axiomas de la lógica clásica. Faculta al pensamiento en sus asociaciones y conexiones de conceptos o enunciados que se contradicen en otro al otro, pero que deben aparecer como dimensiones articuladas de lo mismo. Se precisa que su vocación epistemológica es captar el modo de existencia, el funcionamiento y las interdependencias contextuales de un hecho o situación calificada como compleja (Velilla, 2002).

La dialógica se resume en la advertencia de que en los problemas que surgen en el bucle de orden, desorden y organización, se suele ir en contra de las tesis antagónicas que se plantean como enfrentadas, irreconciliables y excluyentes que devienen en un pensamiento, en extremo simplificador, disyuntor y reductor que subyace en ambas tesis. De modo implícito, al rechazar la parte de verdad que contiene la tesis contraria, cada tesis resulta insuficiente y mutilante. En el contexto de la dialógica se puede desarrollar una visión poliocular.

Finalmente, desde un enfoque de la complejidad el productor del conocimiento está incorporado en el propio conocimiento producido. La universidad es el espacio que alberga de manera natural al método científico como el mejor instrumento que permite crear conocimiento cuando se investiga, y a re-crearlo cuando se enseña. La ciencia progresa porque se evita cometer 
dos veces el mismo error, es como decir que una mosca se estrella contra el cristal y vuelve de nuevo, se estrella otra vez y vuelve, y hasta que muere. Muere en su teoría errónea. Por el contrario el ser humano ha aprendido a hacer morir a sus teorías para no morir él (Antiseri, 1978).

Recuperar al ser humano en el conocimiento que produce lo sitúa no solo como un observador que intenta otear la realidad desde una especie de panóptico, sino que lo hace siendo parte de la misma realidad, pues es producto de ella, porque observa con los ojos que su cultura le provee. La pretensión del principio de reintroducción es en cierta forma bajar a la ciencia desde una poltrona de superiodad para hacerla más modesta, más autocrítica y en consecuencia más empática.

Estas consideraciones descritas podrían, a modo de colofón, mantener la interrogante de si no es el momento de repensar la organización del conocimiento que conduzca a una nueva forma de praxis en el bucle sociedad-universidad-sociedad, dadas las condiciones que avizoran una serie de catástrofes cuyas intensidades ya padece la humanidad. Quedan abiertas todas las posibles respuestas.

\section{Referencias}

Antiseri, Darío (1978). Análisis epistemológico del marxismo y del psicoanálisis. Salamanca: Ediciones Sígueme.

Ardoino, Jacques (2000). La complejidad. En Morin, Unir los conocimientos. La Paz, Bolivia: Plural Editores

Berg, Barbro, et.al. (1977) Innovations and innovate. Processes in higher education. Stockhlm: National Board of Universities and Collages.

Barthes, R (1896). Lo obvio y lo obtuso. Barcelona: Ediciones Paidós.
Bunge (1997). La causalidad, el principio de la causalidad en la ciencia moderna. Buenos Aires: Editorial Sudamericana.

Clark, B.R (1991). El sistema de educación superior. México: Editorial Nueva Imagen.

García, Rolando (2006). Sistemas complejos, conceptos, métodos y fundamentación epistemológica de la investigación interdisciplinaria. Buenos Aires: Gedisa.

Husserl (1975). Ideas relativas a una fenomenología pura y una fenomenología fenomenológica. México: Fondo de Cultura Económica.

Johansen (1986). Introducción a la teoría general de sistemas. México: Editorial LIMUSA, S.A.

Kanizsa, G (1986). Gramática de la visión. Barcelona: Ediciones Paidós.

Kuhn T. (1971). La estructura de las revoluciones científicas. México: Fondo de Cultura Económica.

Maturana y Varela (1990). El árbol del conocimiento. España: Debate.

Moreno (2002). Manual de iniciación pedagógica al pensamiento complejo, Corporación para el pensamiento Complexus, ICFES-UNESCO.

Morin (1992), El método IV. Madrid: Ediciones Cátedra.

Morin (2000). Unir los conocimientos. La Paz, Bolivia: Plural Editores

Morin (2002). La mente bien abierta. Barcelona: Seix Barral, S.A

Nicolescu, Basarab, (1996). La transdisciplinarité. Mónaco: Editions du Rocher. Collection "Transdisciplinarité".

Prigogine (1983). El tiempo y el devenir. Barcelona: Gedisa.

Reynoso, Carlos (2009). Modelos o metáforas- crítica del paradigma de la complejodad según Edgar Morin. Buenos Aires.

Schuldt, Jürgen (2005). ¿Somos pobres porque somos ricos? Lima: Fondo Editorial del Congreso del Perú.

Velilla, M. (2002). Manual de iniciacióm pedagógica al pensamiento complejo. Colombia: UNESCO, Corporación para eñ Desarrollo Complexus.

Vilar, Sergio (1997). La nueva racionalidad: comprender la realidad con métodos transdisciplinarios. Barcelona: Kairós. 